\title{
PHOTO-METRAZOL SENSITIVITY IN CATATONIC SCHIZOPHRENIA
}

\author{
BY \\ D. M. LEIBERMAN and J. HOENIG \\ From the Department of Clinical Neurophysiology, Instititute of Psychiatry,
Maudsley Hospital, London
}

It can be considered as established that epileptic phenomena occur more frequently in catatonic schizophrenia than chance alone would explain. The literature on this subject was summarized by Hill (1948). This increased propensity of the brain of the catatonic patient to discharge in an epileptiform fashion can be assessed quantitatively. The method in use for this purpose, which will be described below, consists in the activation of the E.E.G. by means of intravenous "metrazol" injections combined with simultaneous stimulation of the retina by flickering light. It has been given the portmanteau term "Phomac" (PHOto-Metrazol$A C$ tivation) by Rémond (1952). The origins of this method are as follows. Walter and Walter (1949) have described the use of the electronic stroboscope in the production of paroxysmal E.E.G. changes, myoclonus, or seizures in suitably sensitive subjects. Similarly, intravenous injections of " metrazol" have been used to activate the E.E.G. for diagnostic purposes. The latter method lends itself to a quantitative assessment of the epileptic tendency of the central nervous system (Hutchinson, 1951). Gastaut (1950) combined these two methods. He injected " metrazol" intravenously at a rate of $50 \mathrm{mg}$. every 30 seconds and at the same time stimulated the retina with light flashes from an electronic stroboscope at a rate of 13 to $18 \mathrm{c}$. $/ \mathrm{sec}$. He found that when a certain quantity of " metrazol" had been injected the flash stimulation produced paroxysmal E.E.G. disturbances, myoclonus, or even seizures if pressed far enough. The quantity of " metrazol" required to render the subject sensitive to stroboscopic stimulation of the retina is termed the threshold value, and can be stated in milligrammes of " metrazol", or millilitres of a solution of given strength. The threshold value may vary in different individuals from zero, which means that the subject is sensitive to flicker stimulation alone, to $1,000 \mathrm{mg}$. "metrazol" or over. Values above $1,000 \mathrm{mg}$., however, are uncommon. Following
Rémond, we can term this value the "Phomac threshold ". Among others such as Gastaut (1950), Rémond (1952), Bickford and Daly (1951) we have assessed the Phomac threshold in various diagnostic groups of patients (Leiberman, Hoenig, and Hacker, 1953). We have shown that in catatonic schizophrenics and idiopathic epileptics the mean Phomac threshold is below that of the normal population, a result in substantial agreement with other authors. Other forms of schizophrenia were found to have thresholds in the normal range. We have interpreted the low threshold in catatonic schizophrenics as indicating the tendency of a presumed diencephalic neuronal system to discharge more readily than in normal individuals. This seemed to follow from the explanation of the physiological mechanism of Phomac put forward by Gastaut and Hunter (1950). There are, however, certain observations which seem to indicate that the convulsive threshold varies in each catatonic patient in the course of his illness. For instance, it is well known that some catatonic patients may have epileptiform seizures at certain stages of their illness, most characteristically towards the end of the stupor or shortly after recovery. Hill (1948) found in one patient that at the beginning of a catatonic stupor the amount of paroxysmal spike activity induced by a constant quantity of insulin injected intravenously was at a maximum. This was interpreted to mean that the tendency of the central nervous system to convulse was maximal at this time. Another observation bearing on this subject is that of Corriol and Bert (1950). They reported a case of catatonia in which an epileptiform seizure could be induced by flicker stimulation of the retina at a frequency of about $10 \mathrm{~F}$./sec. During the phase of stupor a short exposure to flicker would induce a seizure, but during remission a considerably longer exposure was necessary.

The present study was carried out in order to investigate possible epileptic threshold variations in catatonic schizophrenia. The investigation consisted 
in the repeated estimation of the Phomac threshold in the course of the illness of five patients.

\section{Method}

The threshold was estimated by the modified Gastaut (1950) method described by Leiberman and others (1953). Patients were asked to attend fasting, i.e. with only a light breakfast, and if they were taking any anticonvulsant drugs were asked to stop taking them for the 24 hours preceding the test. A simple explanation of the procedure was made when possible. An E.E.G. recording was begun, using two fronto-temporal and two temporo-occipital channels. A photo-cell was used in one channel to record the activity of the stroboscope. Such a record was found helpful for the subsequent interpretation. Our technique differs from Gastaut's. A $10 \%$ solution of "metrazol" ("cardiazol ") was injected at the rate of $0.5 \mathrm{ml}$. per 30 seconds. The needle was left in situ during the test. Fifteen seconds after the injection the stroboscope was switched on and off intermittently until the next injection, i.e. for $15 \mathrm{sec}$. at a frequency of 13 to $18 \mathrm{c} . / \mathrm{s}$. During this time the patient opened and closed his eyes as originally described, whenever adequate cooperation made this possible. We believe that the $15 \mathrm{sec}$. delay before the light is flashed allows the "metrazol" time to reach the cerebral circulation, and thus achieves a maximum combined effect. The injection and the stroboscopic flicker were stopped when the threshold, as defined below, was reached or if the patient became incooperative.

Apart from the response most commonly observed by Gastaut, namely myoclonus with frontal polyspikes in the E.E.G., which led him to coin the term " myoclonic threshold", we have observed other responses such as myoclonus with other forms of E.E.G. abnormality, polyspikes without myoclonus, other marked E.E.G. abnormalities without myoclonus, or seizures occurring without E.E.G. warning. We have accepted all these responses as threshold indicators. Reasons for this view are outlined in our report elsewhere (1953). The threshold value was expressed as the total quantity of "metrazol" injected to produce any of the changes described above.

\section{Material}

The examinations were carried out on in-patients of the Bethlem Royal and the Maudsley hospitals. The diagnoses were made by the physicians in charge of the patients. We observed the patients clinically over several months and in some cases a number of recurrent catatonic episodes in hospital were observed. Short clinical histories are given below.

Case 1.-A woman aged 28, suffering from catatonic schizophrenia, was admitted on April 24, 1950, because of recurrence of a catatonic illness. For her first attack she had been treated from March to September, 1949, at another hospital. The condition had responded to electro-convulsive therapy. On present admission she was mute and immobile, showed flexibilitas cerea, was passively obedient though very restricted in her movements, and refused food. She was given 11 electroconvulsive treatments and responded, only to relapse to her previous condition two weeks later. From that stage onward Phomac was used to estimate a threshold repeatedly over the succeeding six months, during which period she was in and out of stupor three times. These remissions were induced by photo-metrazol or electrical convulsions. In many ways she was an ideal subject for the test, as her state of passive obedience made her cooperative and on her the largest number of estimations was made (Fig. 1).
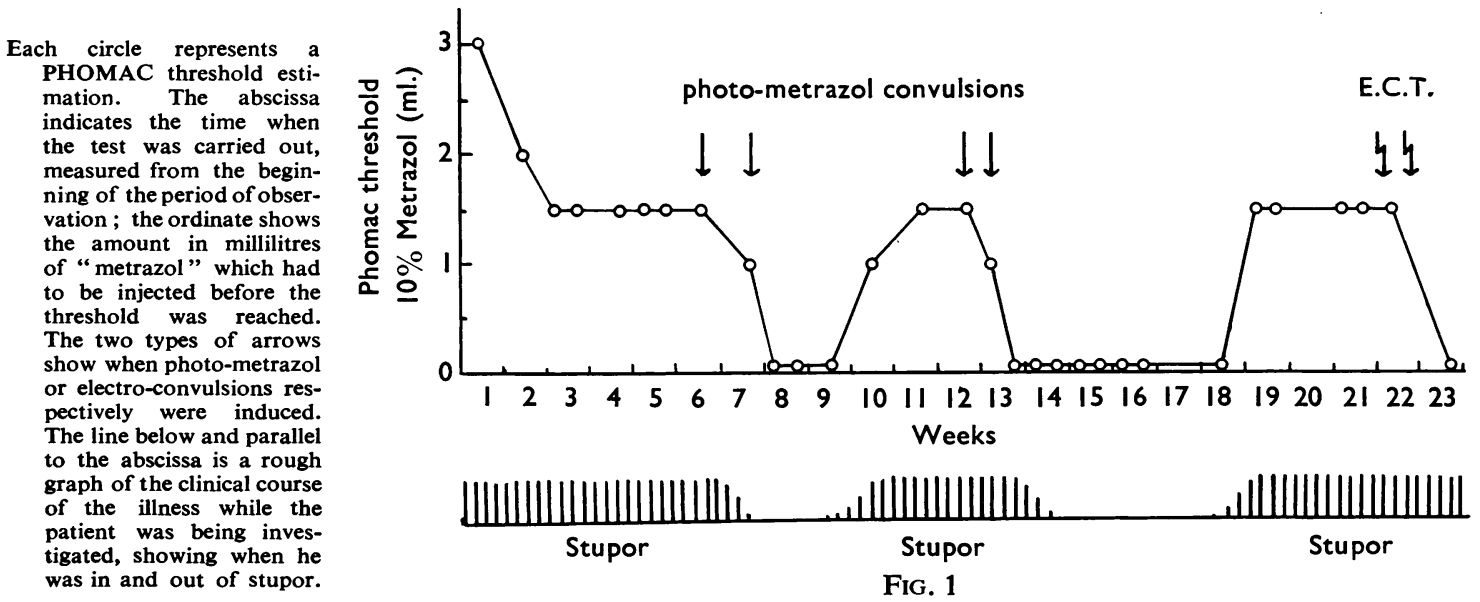
Case 2.-A woman aged 22, suffering from catatonic schizophrenia, was admitted on September 4, 1950, because of a recurrence of a catatonic illness. Her first attack was in September, 1947, and had remitted spontaneously after one month. The next attack occurred in May, 1948, and she was treated successfully with insulin comas and electroconvulsions. After this she remained well for eight months and then had a relapse which lasted only six weeks and ended spontaneously. She remained well until August, 1950. Her present admission was arranged three weeks after the onset of her illness. On admission she was restless and manneristic, showing a number of stereotyped movements. She had thought blocking, was hallucinated, and had a number of hypochondriacal delusions. Her case was followed over some three months. On three occasions a Phomac threshold estimation was made, and then she refused further cooperation in threshold measurements until just before her discharge from hospital. She gave as a reason that the test made her feel strange and frightened (Fig. 2).

Case 3.-A woman aged 29, suffering from catatonic schizophrenia, was admitted on October 3, 1950. She was a woman of shy, retiring personality, who had become increasingly quiet and withdrawn over the past year. Some two months before admission she became restless, continually "turning out" articles of clothing and furniture in her room. Three weeks before admission she became very depressed and agitated. At work she behaved strangely, weeping and repeating to a colleague, "I love John". On admission she was semistuporose, mute and inactive, bewildered, and incontinent of urine and faeces. During the period of ward observation it was noted that at times she appeared hallucinated,
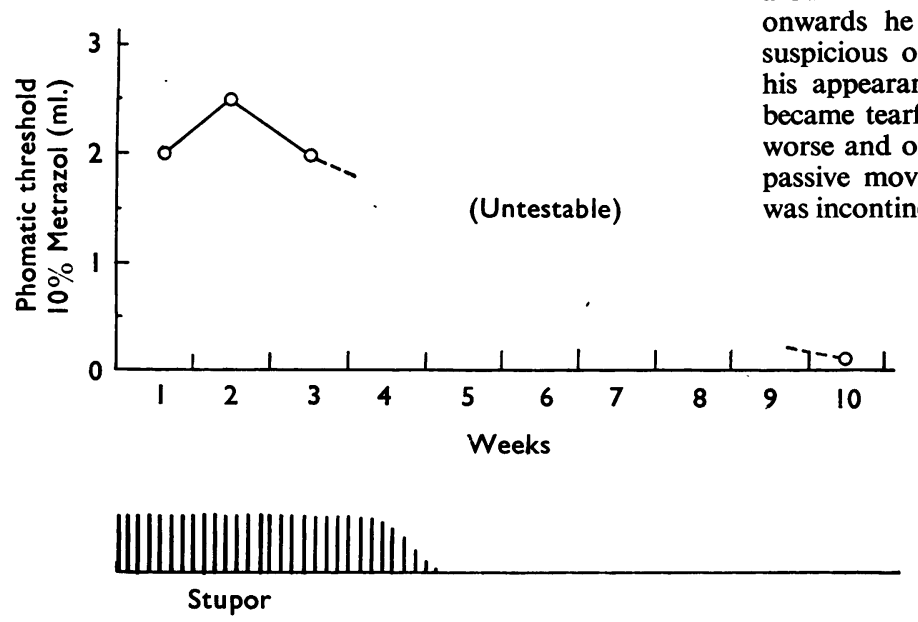

FIG. 2 photo-metrazol convulsions E.C.T.
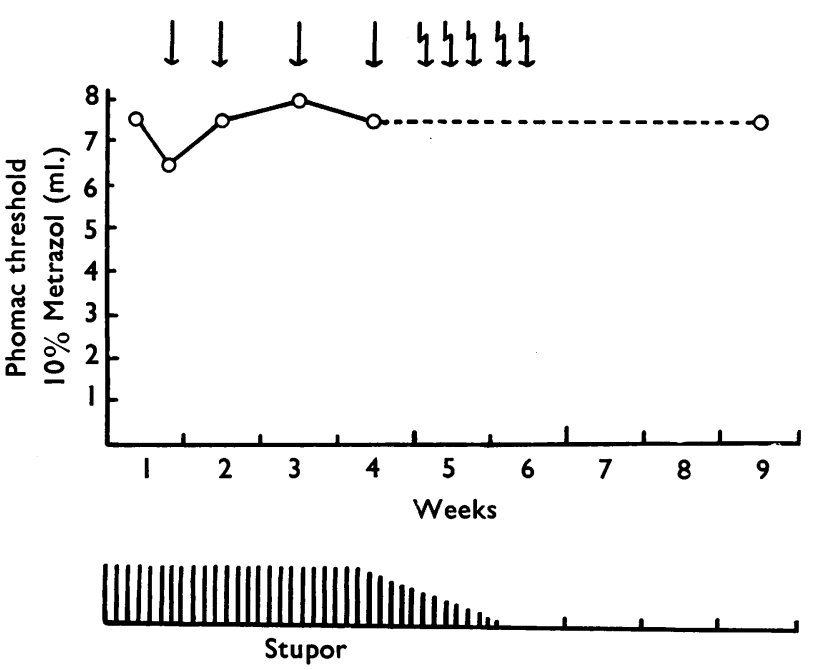

FIG. 3

and when she was induced to speak under the influence of $\dot{\omega}$ " amytal," bizarre delusions were revealed, suggestive $\overrightarrow{0} \overrightarrow{0}$ of schizophrenia. Four photo-metrazol convulsions and $\AA$ five electro-convulsions were given with apparent goo recovery. Phomac thresholds were estimated on severaf occasions (Fig. 3).

Case 4.-A man aged 17, suffering from catatonie schizophrenia, was admitted on December 22,1950 , i catatonic stupor. He had always been a solitary, sensitive person. Although generally unobtrusive he was at times given to outbursts of fierce temper. At school he was said to have been "rather lazy". Six weeks before admission he came home late one evening staggering around in a drooping posture. From that evening onwards he changed progressively. He became very suspicious of people, refused to go out, and neglected his appearance. He felt people were looking at him, me tearful, and slept poorly. He became gradually on admission was found to be mute, resisted passive movements, and showed flexibilitas cerea. He was incontinent and had to be fed and generally cared for. He emerged from the stupor when given electro-convulsive therapy but remained deluded and hallucinated. He was treated with insulin coma but failed to improve further after 34 comas, and on the parents' request was discharged home. Phomac thresholds were estimated before E.C.T. was given (Fig. 4).

Case 5.-A woman aged 18, suffering from recurrent catatonia, was admitted on April 11, 1952, because of " peculiar behaviour" while on night-duty as a student nurse. She had always been 


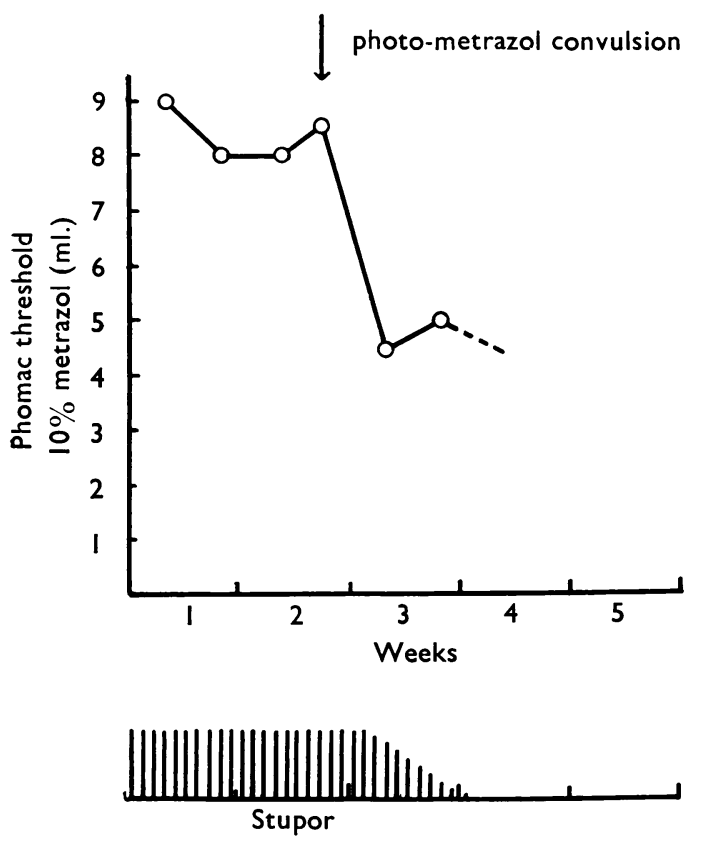

FIG. 4

solitary, selfconscious, and over-conscientious. She had difficulties in making friends, was given to swings of mood and sudden outbursts of temper. At school she was socially poorly adjusted. About two months before admission, following sexual intercourse, she developed irrational fears, and was unable to accept reassurance by her doctor. She appeared preoccupied and her standard of work fell off markedly. She became depressed and irritable and very slow. She had ideas about a trial to be imposed on her by outside

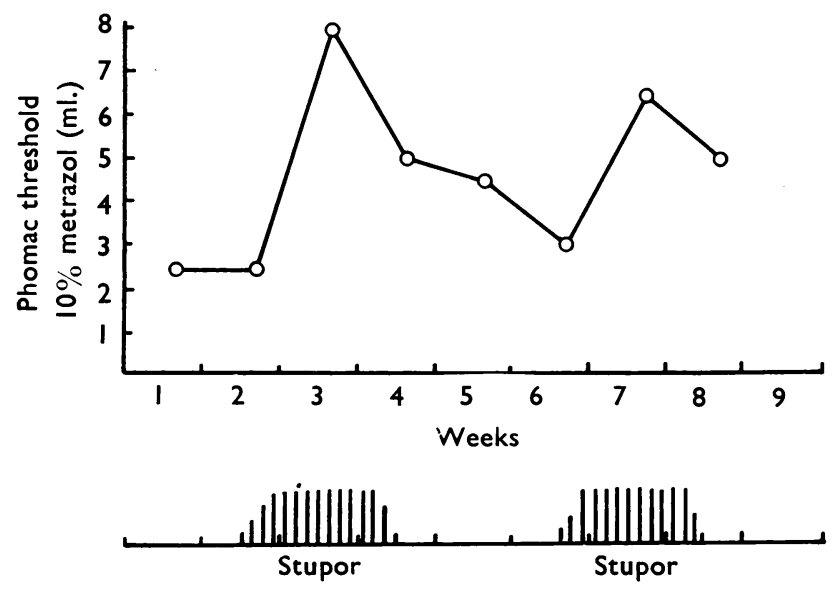

FIG. 5 influences. On admission she was very slow in movement and speech and was depressed. During her stay in hospital she had five clearly delineated periods of disordered behaviour each lasting from 21 to 11 days, getting progressively shorter. Her behaviour during these periods was characterized by social withdrawal, depression, tenseness, and perplexity, which came on suddenly. She refused to talk to anyone and refused food. She resented intrusion and was negativistic. She had self-deprecatory and aggressive ideas. This behaviour subsided again over four to five days and she became bright, friendly, and cooperative for periods of 10 to 20 days. There was no reliable evidence of thought disorder or hallucinations. In view of the somewhat atypical clinical picture a Rorschach test was given which showed obsessional as well as schizophrenic features. Having been free of attacks for two months she was discharged recovered. Phomac tests were carried out at weekly intervals in and out of the attacks (Fig. 5).

\section{Results}

The results are shown graphically in Figs. 1, 2, 3,4 , and 5 , the numbers corresponding to the case number. Except in Case 3 (Fig. 3) the Phomac thresholds show well marked variations parallel to the clinical state of the patient. It seems that when the patient is stuporose, the Phomac threshold is at a maximum, and when the patient is out of stupor or has recovered, the threshold is at a minimum or zero. The patient No. 3 does not fit into this pattern. The reason for this exception is not apparent to us with the data at our disposal. The remissions either occurred spontaneously, or were induced by convulsion therapy, the methods used being either E.C.T. or convulsions induced by photo-metrazol activation.

\section{Discussion}

Before proceeding to discuss possible explanations of these findings, it is necessary to answer the question whether the variations in the threshold could be the result of artefact. For example a false high threshold could be produced by a patient turning his head away. This would prevent the light stimulus from taking effect. It is well known that a stuporose catatonic patient is not the best or most cooperative of subjects, and at times certain of them, notably Case 4 , were most uncooperative, so that the test sometimes had to be abandoned. However, the others (Cases 1, 2, 3, and 5) were generally cooperative so that artefact from this cause can, we think, be discounted. As far as the method is concerned, 
the other variables were kept as constant as possible, and it is unlikely that threshold variations resulted from the method or apparatus.

The next question is whether the threshold variation is specific for catatonic schizophrenia, or whether it is due to non-specific factors which can also be found in other conditions. The clinical state most nearly approaching catatonic stupor is depressive stupor. Here, too, there is a state of prolonged immobility and sluggishness of somatic function. We have estimated the Phomac threshold in patients during depressive stupor and our findings will be reported elsewhere. It was not found to be abnormally low as is often the case in catatonic stupor, nor was it found to vary in the course of the illness.

The variation in the Phomac threshold therefore does not appear to be due either to artefact or to non-specific factors associated with stupor as such, but seems to be caused by some change in the central nervous system which is characteristic of catatonic schizophrenia. There is nothing very unlikely in this. In recurrent catatonia cyclical biochemical changes have been described since Gjessing's $(1932,1938)$ observations on nitrogen metabolism in this condition. More recently the biochemical changes have been linked with cyclical endocrine changes and these in turn with E.E.G. changes (Rowntree and Kay, 1952). .

We may compare the observations in this study with similar observations in the literature (Hill, 1948 ; Corriol and Bert, 1950). The patients in the present series had a relatively low threshold towards the end of stupor and it fell to a lower level or zero when they were in remission. The patient reported by Hill showed a marked tendency to E.E.G. abnormalities when activated by insulin. Hill injected a certain quantity of insulin intravenously on 16 occasions spaced out equally over the course of the illness which lasted 12 weeks. He then assessed the amount of paroxysmal activity in the E.E.G.over a constant period following the injection. He found that the amount of paroxysmal activity was greatest in the beginning and at the end of the stupor, when two spontaneous epileptiform convulsions took place. This finding, if translated into our terminology, would indicate that the patient had a relatively low insulin threshold in the beginning of his illness and just before and immediately after recovering from stupor. Hill's observation would thus seem to be in agreement with the findings in this investigation as far as they are comparable. In comparisons with different activating agents some caution should be observed, since for example, it is known that the response of schizophrenics to insulin may vary from time to time and may be abnormal. The patient of Corriol and Bert was sensitive to flicker, i.e., had a zero Phomac threshold in both stuporose and non-stuporose phases. In the stuporose phase the length of the time that was necessary to produce a convulsion by flicker stimulation was shorter than in the non-stuporose phase. Corriol and Bert concluded that their patient was less liable to convulse in the non-stuporose phase than in the stuporose. The change in convulsive threshold in this case takes place in the opposite direction of that of the authors' patients and of Hill's patient. Though it is not possible to give an explanation of this finding with the data available the following suggests itself. It is known that there are two types of catatonic patients, identified by Gjessing, in which the phases of nitrogen retention and excretion are reversed at comparable clinical phases of the illness. The same may apply to the convulsive threshold; in fact the two kinds of phenomena may be directly related to each other. In one type it may be raised during stupor and low during remission, and in another type the reverse may be the case.

\section{Conclusions}

Since knowledge of the mechanisms in the central nervous system both of Phomac and of catatonic schizophrenia is so meagre, it is not possible to draw firm conclusions about the relationship between variations in threshold and the clinical state. Nevertheless the authors believe that the observations in themselves are sufficiently interesting to be brought to the notice of other workers.

This study reaffirms previous findings reported in the literature that the Phomac threshold in catatonic schizophrenia is lower than that of the normal population. It has to be pointed out, however, that this refers only to statistical means and that there is considerable individual variation. Although Cases 4 and 5 do not show a low threshold during stupor, it does undoubtedly fall below normal during a remission. Even more interesting is the variation in the threshold during the course of the illness. The lowered threshold in non-stuporose stages of the illness is interpreted to mean that there is an increased tendency to discharge on the part of a central neuronal system when the patient emerges from stupor. Gastaut (1950) has suggested that the low Phomac threshold indicates a functional diencephalic disturbance. It would appear that in the schizophrenic patients in this study restitution of normal function of the central nervous system, i.e., emergence from stupor, is associated with a lowering of the Phomac threshold; that is to say 
there is an increased tendency for a diencephalic mechanism to discharge. Although no direct evidence for such a view is at present in existence, it is very likely that a dynamic relationship exists between this tendency to discharge, as measured by the Phomac threshold, and the clinical state. The patient will emerge from stupor if a certain low threshold is reached and maintained. This hypothesis appears in agreement with the conclusions of Gellhorn (1943) and others who studied the changes in excitability in an autonomic regulating centre following successful physical treatment of schizophrenia. They deduced such changes from observation of alterations in the function of the autonomic nervous system. Gellhorn concluded on the basis of his own work and that of others that in schizophrenia there is a variation in the reactivity or excitability of a central mechanism which functions through the sympathetico-adrenaland vagoinsulin systems. Hill and others (1951) have produced evidence of a like nature. Both Gellhorn and Hill suggest that this central mechanism is a diencephalic autonomic centre. Gellhorn further suggests that the effect of physical treatment in schizophrenia is brought about by a stimulation of this centre, and a consequent increased excitability of the sympathetico-adrenal system. In this connexion it is interesting to note that in our series the threshold varies in the same manner no matter whether the remission occurs spontaneously as in Cases 2 and 5 , or whether it is induced by convulsive therapy as in Cases 1 and 4. A mechanism of this sort may have a homeostatic function in relation to the rest of the central nervous system (Hill, 1952), and the cyclical endocrine and metabolic changes observed in catatonic schizophrenia may be mediated through such a system.

\section{Summary}

The photo-metrazol threshold has been assessed repeatedly at different times in the course of the illness in five patients suffering from catatonic schizophrenia. The variations thus observed were related to the changing clinical state.

In four patients the threshold fell as they emerged from stupor.

It is suggested that the fall in threshold represents a rise in the excitability of a presumed diencephalic homeostatic mechanism; which can occur either spontaneously or be brought about by convulsion therapy.

We wish to express our thanks to Dr. Denis Hill for his constant encouragement and advice, and also for his permission to carry out the investigation in his laboratory.

\section{REFERENCES}

Bickford, R. G., and Daly, D. (1951). Electroenceph. clin. Neurcphysicl., 3, 378 .

Corriol, J., and Bert, J. (1950). Ann. Méd-Psychol., 108, 588.

Gastaut, H. (1950). Ibid., 2, 249.

$\overline{\text {, }}$, and Hunter, J. (1950). Ibid., 2, 263.

Gellhorn, E. (1943). Autonomic Regulations. Interscience Publishers Inc: New York.

Gjessing, R. (1932). Arch. Psychiat., 96, 319.

(1938). J. ment. Sci., 84, 608.

Hill, D. (1948). Folia psychiat., Amst., 51, 95. (Congress number.) (1950). E.E.G. in Psychiatry, Comptes rendus 1st Internat. 1950). E.E.G. in Psychiatry, Comptes rendus
Congress Psychiatry, Paris, vol. 3, pp. 164-178.

Congress Psychiatry, Paris, vol. 3, pp. 164-178.
(1952). Electroenceph. clin. Neurophysiol., 4, 419.

- (1952). Electroenceph. clin. Neurophysiol., 4, 419. Sci., 97,111

Hutchinson, J. T. (1951). Proc. roy. Soc. Med., 44, 325.

Leiberman, D. M., Hoenig, J., and Hacker, M. (1953). "The Cardiazol Flicker Threshold in 104 Neuropsychiatric Patients." diazol Flicker Threshold in 104 Neuropsychiatr
Electroenceph. clin. Neurophysiol. In the Press.

Electroenceph. clin. Neuroph
Rémond, A..(1952). Ibid., 4, 265.

Rowntree, D. W., and Kay, W. W. (1952). J. ment. Sci., 98, 100

Walter, V. J., and Walter, W. Grey (1949). Electroenceph. clin. 